\title{
Server load prediction algorithm based on CM-MC for cloud systems
}

\author{
XU Xiaolong ${ }^{1, *}$, ZHANG Qitong $^{1,2}$, MOU Yiqi $^{3}$, and LU Xinyuan ${ }^{4}$ \\ 1. Jiangsu Key Laboratory of Big Data Security \& Intelligent Processing, Nanjing University of Posts and Telecommunications, \\ Nanjing 210023, China; \\ 2. State Key Laboratory of Information Security, Chinese Academy of Sciences, Beijing 100093, China; \\ 3. Shanghai Stock Exchange Technology Co., Ltd, Shanghai Stock Exchange, Shanghai 200120, China; \\ 4. Institute of Big Data Research at Yancheng, Nanjing University of Posts and Telecommunications, Yancheng 224000, China
}

\begin{abstract}
Accurate prediction of server load is important to cloud systems for improving the resource utilization, reducing the energy consumption and guaranteeing the quality of service (QoS). This paper analyzes the features of cloud server load and the advantages and disadvantages of typical server load prediction algorithms, integrates the cloud model (CM) and the Markov chain (MC) together to realize a new CM-MC algorithm, and then proposes a new server load prediction algorithm based on CM-MC for cloud systems. The algorithm utilizes the historical data sample training method of the cloud model, and utilizes the Markov prediction theory to obtain the membership degree vector, based on which the weighted sum of the predicted values is used for the cloud model. The experiments show that the proposed prediction algorithm has higher prediction accuracy than other typical server load prediction algorithms, especially if the data has significant volatility. The proposed server load prediction algorithm based on CM-MC is suitable for cloud systems, and can help to reduce the energy consumption of cloud data centers.
\end{abstract}

Keywords: cloud computing, load prediction, cloud model, Markov chain, energy saving.

DOI: $10.21629 /$ JSEE.2018.05.17

\section{Introduction}

The accurate prediction of server load is of great significance for cloud system to improve the resource utilization, reduce the energy consumption and guarantee the quality of service (QoS) [1-5]. Based on the right results of server load prediction, virtual machine (VM) can be deployed and migrated more orderly, avoiding the unwanted virtual machine migrations, the network traffic, and the transient

Manuscript received September 11, 2017.

*Corresponding author.

This work was supported by the National Natural Science Foundation of China (61472192; 61772286), the National Key Research and Development Program of China (2018YFB1003700), the Scientific and Technological Support Project (Society) of Jiangsu Province (BE2016776), and the "333" Project of Jiangsu Province (BRA2017228; BRA2017401). spikes of load peak [6]. Besides, load prediction can also extend the life of the hardware equipment and reduce the energy consumption of the cloud data center under the assurance of the QoS and the service-level agreement (SLA) [7].

The server load of the cloud data center, e.g., the CPU load, has the following features [8]:

(i) Nonlinearity. The load monitoring data cannot be fitted with a linear function, and the data has the uncertainty.

(ii) Strong volatility. Volatility is a statistical indicator, referring to the degree of a series of load monitoring data rising or falling in a short period of time.

Due to the non-linearity and the strong volatility of server load, it is difficult to ensure the accuracy of the load prediction algorithm and reduce its cost at the same time [9].

The current load prediction schemes includes nonheuristic algorithms [1,10-12] and heuristic algorithms $[2,5-8]$. However, they generally have the following issues to be solved:

(i) Non-heuristic algorithms have lower prediction accuracy and are not suitable for the situation with strong volatility.

(ii) The heuristic algorithms have higher computational cost, which is not suitable for systems with the strict realtime requirement.

(iii) The time granularity and the target granularity of current load prediction algorithms are not fine enough. Some load prediction algorithms predict the overall system load in the unit of day in order to provide the basis for adjusting the number of running servers, which can save limited energy consumption.

In this paper, we propose a new server load prediction algorithm. The main contributions of this paper are as follows: 
(i) We integrate the cloud model (CM) and the Markov chain (MC) together to realize a new CM-MC algorithm. The CM-MC algorithm inherits the historical data sample training method of the CM. Each cloud in the CM is a state, and the Markov initial matrix is established with the membership grade of the current data to the cloud. Then, the Markov transition matrix is established based on the state transition of historical data to obtain the predicted value with the membership weighting method.

(ii) We propose a new server load prediction algorithm based on CM-MC for cloud systems. For the multi-type load prediction, in the proposed algorithm we design a multi-dimensional method, which uses multiple variables to construct the state transition matrix to carry out the load prediction.

\section{Related works}

Current non-heuristic load prediction algorithms are based on the MC, the gray model, the autoregressive integrated moving average (ARIMA) model, or the exponential smoothing method respectively, etc. Muñoz-Escoí et al. [10] established the multiple sub-models and then use the autoregressive method to predict the future load for preparing the required virtual machines in advance. However, this method brings too much cost, and can only be used for the virtual machine allocation. Li et al. [11] used a multitimeline collaborative load prediction algorithm for cloud systems. This algorithm uses the hidden Markov model to mine the dependencies between applications running on different virtual machines to classify virtual machine types and then manage the platform through the relevance. The algorithm is more suitable for the cloud system with large-scale virtual machines providing the same type of services. However, its time granularity of prediction is not fine enough. Hao et al. [12] used the ARIMA algorithm for load forecasting to allocate resources and release unnecessary resources in advance, aiming to improve the QoS of cloud system. The prediction results of this algorithm quite match the real conditions of cloud system, while its prediction accuracy of the peak load is not good enough.

The main idea of load prediction based on the heuristic algorithm is to obtain the forecasting model by learning and training the historical data, and then input the current value to the model to get the predicted value. Barati et al. [2] improved the tuned support vector regression (TSVR) algorithm, which prepares the vector data to improve the prediction accuracy and avoid the premature convergence. However, the improved algorithm needs to take a long time to select the appropriate SVR parameters. Zhang [7] proposed a load prediction algorithm based on the back propagation neural network to obtain the ideal performance.
However, the computing cost of the algorithm is high. Liu [5] proposed the load prediction algorithm based on the $\mathrm{CM}$, which extracts large-scale historical data to obtain a cloud function set (i.e., a CM), and obtain the load prediction value with the sprinkling method. The $\mathrm{CM}$ has been proved to be scalable [13]. Zhang [5] optimized the construction process of the CM. Compared with other prediction algorithms, the current prediction algorithms based on the CM has better performance in the mass. However, the prediction results with the $\mathrm{CM}$ are highly volatile and there is still room for improvement in the prediction accuracy. What is more, the number of iterations is too large and the computational cost is very high.

Cao [14] pointed out that the prediction accuracy will be relatively low with single element of time sequence, while on cloud systems it is necessary to consider the correlation between different parameters to improve the prediction accuracy. Hameed [15] pointed out that the load prediction should be considered comprehensively with CPU, storage and network, and the prediction should be able to cope with the drastic change of load.

\section{Section preliminaries}

\subsection{Markov process}

Definition 1 Markov process [16]. The Markov process is named after the Russian mathematician Andrey Markov, which refers to a stochastic process with the non-aftereffect property which can be used for the prediction of discrete time series. The non-aftereffect property means that the state of system at the moment $t_{0}$ can determine the state of the system or process at the moment $t>t_{0}$ without relying on previous states of system before $t_{0}$.

Definition 2 MC [17]. The MC is a type of Markov process that has either discrete state space or discrete index set. The MC is defined as a stochastic process $\{X(t), t \in$ $T\}$ with the state space $I$. Supposing that there are $n$ values, $n \geqslant 3, X\left(t_{i}\right)=x_{i}, x_{i} \in I, i=1,2, \ldots, n-1$, the conditional distribution function of $X\left(t_{i}\right)$ is defined as

$$
\begin{gathered}
P\left\{X\left(t_{n}\right) \leqslant x_{n} \mid X\left(t_{1}\right)=x_{1},\right. \\
\left.X\left(t_{2}\right)=x_{2}, \ldots, X\left(t_{n-1}\right)=x_{n-1}\right\}= \\
P\left\{X\left(t_{n}\right) \leqslant x_{n} \mid X\left(t_{n-1}\right)=x_{n-1}\right\}, \quad x_{n} \in \mathbf{R} .
\end{gathered}
$$

\section{$3.2 \mathrm{CM}$}

Randomness and fuzziness are the two most important uncertainties inherent in human cognition, which have attracted great attention in artificial intelligence research. Regarding linguistic terms or concepts as the basic units of human cognition, Li et al. [18] proposed a new cognitive model, the CM, which can synthetically describe the 
randomness and fuzziness of concepts and implement the uncertain transformation between a qualitative concept and its quantitative instantiations. The advantage of the prediction algorithm based on the CM is the feature of uncertainty, more adaptive for the uncertainty description of linguistic concepts.

Definition 3 Cloud and cloud drop [19]. Suppose that $U$ is a quantitative domain, $C$ is the qualitative concept on $U$, and $X$ is a random variable defined on $U$ with the random implementation $x$. The membership grade $x$ to $C$, $\mu_{C}(x) \in[0,1]$, is random while tending to be stable. The distribution of $X$ on $U$ is called the cloud, and $x$ is called the cloud drop.

A typical cloud is shown in Fig. 1(a), where each point is a cloud drop. A cloud can be expressed as $c(E x, E n, H e)$. $E x$ is the mathematical expectation of the cloud, i.e., the most possible value describing the qualitative concept in the cloud.

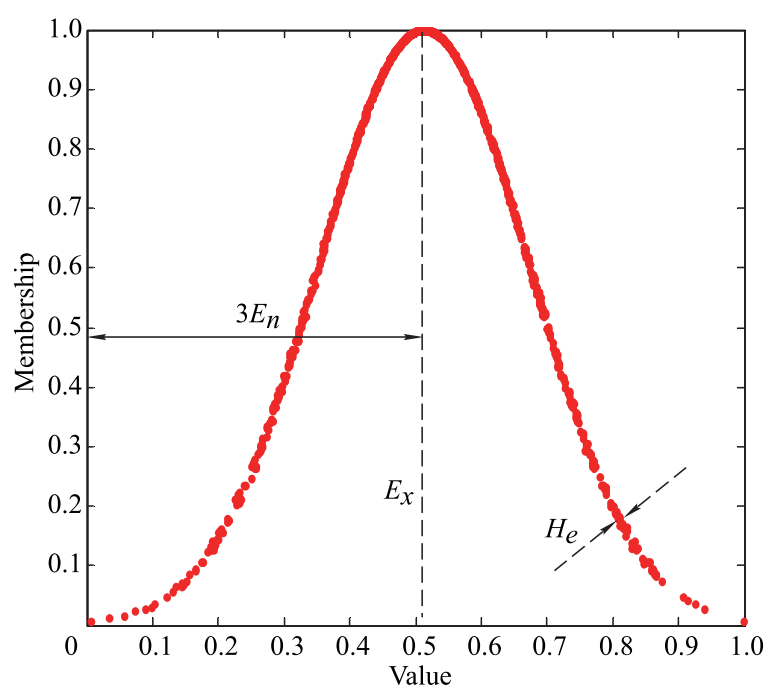

(a) A cloud

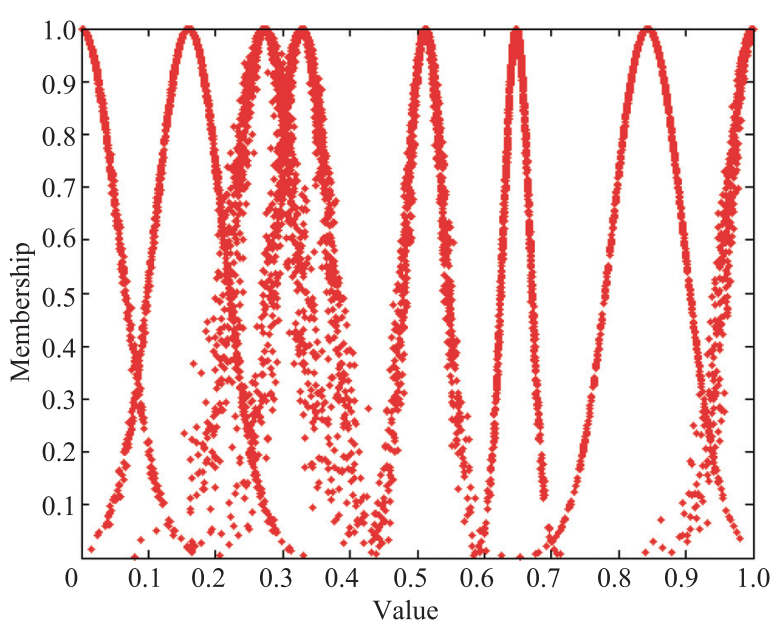

(b) $\mathrm{CM}$

Fig. 1 A cloud and CM
$E n$ is the entropy of the cloud, representing the degree of discretization of the mathematical expectation. $\mathrm{He}$ is the super entropy of the cloud, i.e., the degree of discrete entropy. The larger the fluctuation range of the prediction set, the greater the ambiguity, which can be expressed by the entropy. The randomness of the predicted result is determined by the super entropy. The set of clouds described for the whole data is called the CM, which is shown in Fig. 1(b).

The function for generating cloud drops and CM is called the cloud generator, including the forward cloud generator and the backward cloud generator. The prediction workflow based on the CM is shown in Fig. 2. The key information of the historical data is extracted by the backward cloud generator, and the set of CM is obtained; and then, the right $\mathrm{CM}$ is selected according to the current data; finally, the prediction value is obtained with the forward cloud generator.

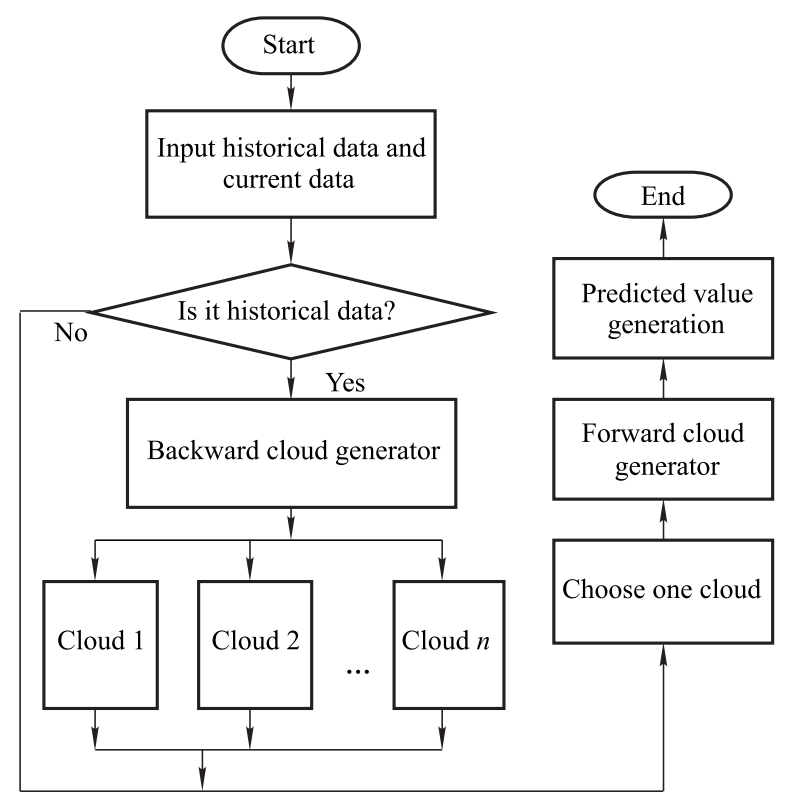

Fig. 2 Prediction workflow based on the CM

Definition 4 Forward cloud generator. The forward cloud generator is used to generate point $\{x, \mu\}$ based on the features of the CM, so as to obtain the prediction value.

The membership grade function of the forward cloud generator is defined as

$$
\gamma(x)=\mathrm{e}^{\left(-\frac{\left(x-E_{x}\right)^{2}}{2 E_{n}^{2}}\right)} .
$$

Definition 5 Backward cloud generator. The backward cloud generator is used to determine mathematical expectations, entropies, and super entropies from a significant number of cloud drops.

The backward cloud generator completes the change from the quantitative number to qualitative concept. The 
original data is normalized, fitted, changed, merged to get the qualitative concept, so as to establish the CM backward. Most backward establishments of cloud models use the peak to peak method [19] to realize fitting. The process of extracting primitive information of the $\mathrm{CM}$ is as follows [20]:

Step 1 Calculate the sample mean $\bar{X}=\frac{1}{n} \sum_{i=1}^{n} x_{i}$, the first order sample absolute center distance $\bar{X}=\frac{1}{n} \sum_{i=1}^{n} x_{i}$, and the sample variance $S^{2}=\frac{1}{n-1} \sum_{i=1}^{n}\left(x_{i}-\bar{X}\right)^{2}$;

Step 2 Search for the locations of peaks in the frequency distribution statistics of the load data, and define the corresponding abscissa as the expectation of the CM;

Step 3 Calculate the entropy $E n=\sqrt{\frac{\pi}{2}} \times \frac{1}{n} \sum_{i=1}^{n} \mid x_{i}-$ $E x \mid$;

Step 4 Calculate the super entropy $H e=\sqrt{S^{2}-E n^{2}}$.

The establishment of the CM encounters the problem of similarity, that is, the overlap between the two clouds is too large. Therefore, for a preliminary establishment of the $\mathrm{CM}$, it is necessary to compare and merge similar CMs by computing the proximity and the overlap degree of the two clouds.

Definition $63 E n$ principle of normal cloud. The contribution of cloud drops in domain $U$ to the concept of $C$ is mainly located in $[E x-3 E n, E x+3 E n]$, and the contribution outside $[E x-3 E n, E x+3 E n]$ is negligible, as shown in Table 1 [21].

Table 1 Contributions of cloud drops to the concept $C$

\begin{tabular}{|c|c|c|}
\hline Interval & $\begin{array}{l}\text { Ratio of the } \\
\text { cloud drops to } \\
\text { all cloud drops } / \%\end{array}$ & $\begin{array}{l}\text { Ratio of the } \\
\text { contribution to } \\
\text { all contributions/\% }\end{array}$ \\
\hline$[E x-0.67 E n, E x+0.67 E n]$ & 22.33 & 50 \\
\hline$[E x-E n, E x+E n]$ & 33.33 & 68.26 \\
\hline $\begin{array}{c}{[E x-2 E n, E x-E n] \mathrm{U}} \\
{[E x+E n, E x+2 E n]}\end{array}$ & 33.33 & 27.18 \\
\hline $\begin{array}{c}{[E x-3 E n, E x-2 E n] \mathrm{U}} \\
{[E x+2 E n, E x+3 E n]}\end{array}$ & 33.33 & 3 \\
\hline
\end{tabular}

\section{Prediction algorithm based on CM-MC}

\subsection{Workflow of algorithm}

The CM establishment approach of CM-MC is based on the basic backward cloud generator, while creatively proposes to improve the load prediction mechanism by integrating the Markovian transfer matrix. The workflow of the server load prediction algorithm based on CM-MC for cloud systems is shown in Fig. 3.

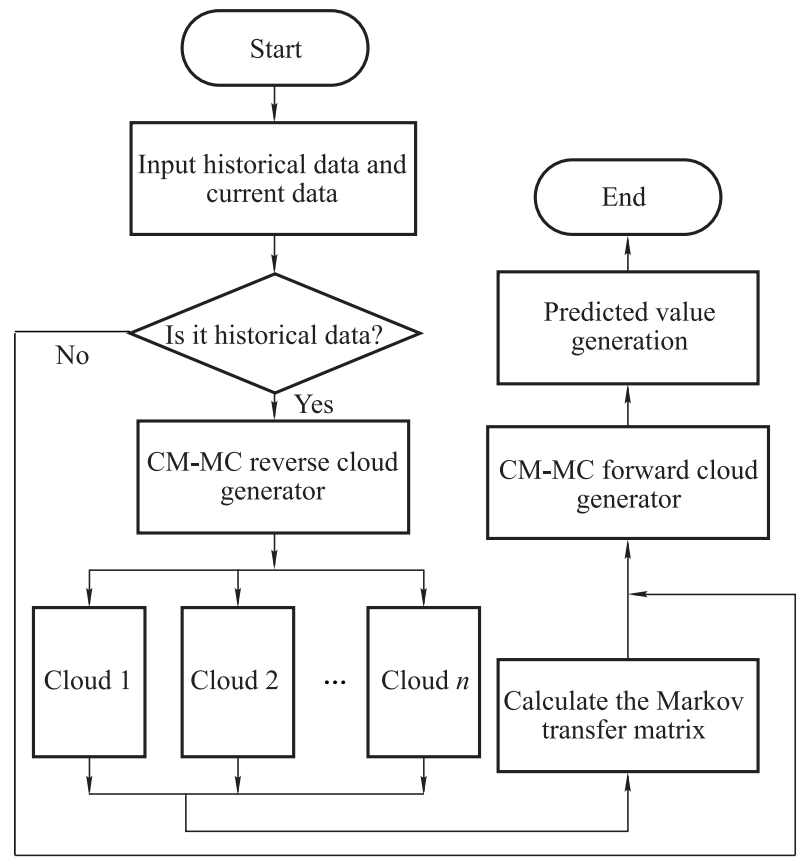

Fig. 3 Workflow of the prediction algorithm based on CM-MC

Step 1 The CM is trained with historical data.

First of all, the data needs to be pre-processed, especially normalized. Set the original historical sequence of the predicted object, $\left\{a_{1}, a_{2}, \ldots, a_{q}\right\}$, where the maximum value is $a_{\max }$ and the minimum value is $a_{\min }$.

In order to obtain the normalized data set $\left\{x_{1}, x_{2}, \ldots, x_{q}\right\}$, we apply the following normalization method to CM-MC:

$$
x_{i}=\left(x_{i}-a_{\min }\right) /\left(a_{\max }-a_{\min }\right) .
$$

The distribution function $f(x)$ is obtained via processing $\left\{x_{1}, x_{2}, \ldots, x_{q}\right\}$. Search the peak and valley locations of $f(x)$ and divide $f(x)$ into several sub-functions, $\left\{z_{1}(x), z_{2}(x), \ldots, z_{n}(x)\right\}$, by every two peaks and valleys for a section. Therefore, the original CMs are obtained, $h\left\{h\left(E x_{1}, E n_{1}, H e_{1}\right), h\left(E x_{2}, E n_{2}, H e_{2}\right), \ldots, h\left(E x_{n}\right.\right.$, $\left.\left.E n_{n}, H e_{n}\right)\right\}$. Two clouds with too large overlapping areas are merged to get the CM set $\left\{c\left(E x_{1}, E n_{1}, H e_{1}\right)\right.$, $\left.c\left(E x_{2}, E n_{2}, H e_{2}\right), \ldots, c\left(E x_{m}, E n_{m}, H e_{m}\right)\right\}$. About the clouds' combination approaches $[5,22,23]$, we use the overlap determination method [5] to calculate the ratio of overlap, that is,

$$
\left\{\begin{array}{l}
E_{x}=\frac{E_{x 1} E_{n 1}^{\prime}+E_{x 2} E_{n 2}^{\prime}}{E_{n 1}^{\prime}+E_{n 2}^{\prime}} \\
E_{n}=E_{n 1}^{\prime}+E_{n 2}^{\prime} \\
H_{e}=\frac{H_{e 1} E_{n 1}^{\prime}+H_{e 2} E_{n 2}^{\prime}}{E_{n 1}^{\prime}+E_{n 2}^{\prime}}
\end{array} .\right.
$$

Step 2 The Markov transfer matrix is obtained. 
Each data form normalized data set $\left\{x_{1}, x_{2}, \ldots, x_{t}\right\}$ for historical datasets can obtain the membership degree for each CM. The degree of membership is calculated with

$$
\mu(x)=\mathrm{e}^{\left(-\frac{\left(x-E_{x}\right)^{2}}{2 E_{n}^{2}}\right)} .
$$

The membership set $\left\{\mu\left(x_{i}\right)_{1}, \mu\left(x_{i}\right)_{2}, \ldots, \mu\left(x_{i}\right)_{n}\right\}$ of $x_{i}$ is got based on the historical CMs $\left\{c\left(E x_{1}, E n_{1}, H e_{1}\right)\right.$, $\left.c\left(E x_{2}, E n_{2}, H e_{2}\right), \ldots, c\left(E x_{m}, E n_{m}, H e_{m}\right)\right\}$. Each CM can be taken as a state. Supposing that the state at time $m$ is $a_{i}$, the probability of transferring to $a_{j}$ at time $m+n$ is set as

$$
\boldsymbol{P}_{i j}(m, m+n)=\boldsymbol{P}\left\{X_{m+n}=a_{j} \mid X_{m}=a_{i}\right\} .
$$

$\boldsymbol{P}(m, m+n)=\left(\boldsymbol{P}_{i j}(m, m+n)\right)$ is the state transition probability matrix of the MC.

Step 3 The membership of the current data for each $\mathrm{CM}$ is calculated.

Suppose there is $\left\{a_{t-1}, a_{t}\right\}$, and $a_{t}$ is at the current time $t$ and $a_{t-1}$ is at time $t-1 .\left\{a_{t-1}, a_{t}\right\}$ is normalized to get $\left\{x_{t-1}, x_{t}\right\} . x_{t}$ is calculated with (5) to get the membership vector $\left(\mu\left(x_{t}\right)_{1}, \mu\left(x_{t}\right)_{2}, \ldots, \mu\left(x_{t}\right)_{n}\right)$.

Step 4 The prediction value is obtained by the CM-MC generator.

As the $3 E n$ principle requires, $\left(\mu\left(x_{t}\right)_{1}, \mu\left(x_{t}\right)_{2}, \ldots\right.$, $\left.\mu\left(x_{t}\right)_{n}\right)$ needs to be screened first. Those points with their expected values in $[-\infty, E x-3 E n] \cup[E x+3 E n,+\infty]$ is negligible. For $c(E x, E n, H e)$, the marginal condition membership value is

$$
\mu_{\text {limit }}=\mathrm{e}^{\left(-\frac{\left(E x-3 E n-E_{x}\right)^{2}}{2 E_{n}^{2}}\right)}=\mathrm{e}^{\left(-\frac{\left(E x+3 E n-E_{x}\right)^{2}}{2 E_{n}^{2}}\right)}=
$$

$$
0.011108997 \text {. }
$$

The data in the membership vector is set 0 . If it is below $\mu_{\text {limit }}$, calculate a new membership vector $\left(\mu^{\prime}\left(x_{t}\right)_{1}, \mu^{\prime}\left(x_{t}\right)_{2}, \ldots, \mu^{\prime}\left(x_{t}\right)_{n}\right)$. If all data is below $\mu_{\text {limit }}$, then the highest value will be retained. $\left(\mu^{\prime}\left(x_{t}\right)_{1}, \mu^{\prime}\left(x_{t}\right)_{2}, \ldots, \mu^{\prime}\left(x_{t}\right)_{n}\right)$ is set as the initial matrix $\boldsymbol{P}(0)$ to get $\boldsymbol{P}(n)=\boldsymbol{P}(0) \boldsymbol{P}(n)$. For each cloud $C(E n, E x, H e)$, a normal random number $y$ with $E n$ as expectation and $\mathrm{He}$ as entropy is generated. The membership of the random number to the cloud is in accordance with the $3 E n$ principle. According to the measured data of the previous step to determine, if it shows the rising trend, $y^{\prime}=E n+\sqrt{-2 \ln x} \times y$; if not, $y^{\prime}=E n-\sqrt{-2 \ln x} \times y$. The prediction sequence $\left\{y_{1}^{\prime}, y_{2}^{\prime}, \ldots, y_{n}^{\prime}\right\}$ is obtained, and the final prediction value is the weighted average of $y^{\prime}$ and $\mu^{\prime}$.

\subsection{Pseudo-code}

Suppose that $\left\{a_{1}, a_{2}, \ldots, a_{t}\right\}$ is the set of historical data for the predicted object, and $\left\{C\left(E x_{i}, E n_{i}, H e_{i}\right)(i=\right.$
$1,2, \ldots, n)\}$ is the outputted CM. The pseudo-code of establishing the $\mathrm{CM}$ is as follows.

Input: $\left\{a_{1}, a_{2}, \ldots, a_{t}\right\}$

Output: CM series $\left\{C\left(E x_{i}, E n_{i}, H e_{i}\right)(i=1,2, \ldots\right.$, $n)\}$; Markov transfer matrix $P(n)$

1) Set $n=$ num of the inputed time series

2) While $(n)$

3) Get $x_{i}=x_{i}-a_{\min } /\left(a_{\max }-a_{\min }\right) \quad / * i=1,2,3$, $4, \ldots, n * /$

4) End While

5) $h(x)=\operatorname{Split}(\{x\}) \quad / *$ Cut the sequence $\{x\}$ according to the peak method $* 1$

6) Set $m=\operatorname{num}$ of $h(x)$

7) While $(m)$

8) $\quad$ Set $l=\operatorname{num}$ of $h(x) i \quad l * i=1,2,3,4, \ldots, m * /$

9) $\operatorname{Get} \bar{X}_{i}=\frac{1}{l} \sum_{j=1}^{l} x_{j} \quad I^{*} j=1,2,3,4, \ldots, l * /$

10) $\quad$ Get $S_{i}^{2}=\frac{1}{l-1} \sum_{j=1}^{l}\left|x_{j}-\bar{X}\right|^{2}$

11) $\quad$ Get $E x_{i}=\frac{1}{l} \sum_{j=1}^{l}\left|x_{j}-\bar{X}\right|$

12) Get $E n_{i}=\sqrt{\frac{\pi}{2}} \times \frac{1}{l} \sum_{j=1}^{l}\left|x_{j}-E x\right|$

13) Get $H e_{i}=\sqrt{S_{i}^{2}-E n_{i}^{2}}$

14) Get $h_{i}\left(E x_{1}, E n_{1}, H e_{i}\right)$

15) End While

16) Get $\left\{C\left(E x_{i}, E n_{i}, H e_{i}\right)(i=1,2, \ldots, n)\right\}$ by overlap $(\{h(x)\}) \quad / *$ Merge the CM */

17) Get $P(n)=\operatorname{Markov}\left\{\left(t_{1}, a_{1}\right),\left(t_{2}, a_{2}\right), \ldots,\left(t_{n}, a_{n}\right)\right\}$ /* Get Markovian transfer statistics from the time series */ $\left\{C\left(E x_{i}, E n_{i}, H e_{i}\right)(i=1,2, \ldots, n)\right\}$ and the time sequence $\left\{a_{t-1}, a_{t}\right\}$ are used as the inputs. $a_{t}$ indicates the current data and $a_{t-1}$ indicates the data at the previous time point; $a_{\max }$ and $a_{\min }$ represent the maximum and minimum values in the history training samples. Base on the Markov transfer matrix $\boldsymbol{P}(n)$, the algorithm will predict the value $a_{t+1}$. The pseudo-code is as follows.

Input: $\mathrm{CM}$ series $\left\{C\left(E x_{i}, E n_{i}, H e_{i}\right) \quad(i=1\right.$, $2, \ldots, n)\} ;\left\{a_{t-1}, a_{t}\right\}$; Markov transfer matrix $\boldsymbol{P}(n)=$ $\boldsymbol{J}\left\{J_{1}, J_{2}, \ldots, J_{n}\right\}$

Initialize: int $a_{t+1}=0$; int $y=0$

Output: Predictive value $a_{t+1}$

1) Set length $=n$

2) While $(n)$

3) $\operatorname{Get} \mu_{i}=\mathrm{e}^{\left(-\left(\frac{x_{i}-E x_{i}}{2 E n_{i}}\right)^{2}\right)} / * i=1,2,3,4, \ldots, n * /$

4) End While

5) Get $\boldsymbol{P}(0)=\left(\mu_{1}, \mu_{2}, \ldots, \mu_{n}\right)$

6) Get $\boldsymbol{P}(n)=\boldsymbol{P}(0) \boldsymbol{P}(n)=\left(\mu_{1}^{\prime}, \mu_{2}^{\prime}, \ldots, \mu_{n}^{\prime}\right)$ 
7) While $(n)$

8) If $\left(\mu_{i}^{\prime}>\mu_{\text {limit }}\right) / * i=1,2,3,4, \ldots, n * /$

9) Get $x_{i}=$ normrnd $\left(E n_{i}, H e_{i}\right) / *$ Generate a normal random number with expectation $E n_{i}$ of entropy $H e_{i} * /$

15)

19)

$$
\text { If }\left(a_{t}<a_{t-1}\right)
$$$$
\text { Get } y_{i}^{\prime}=E n-\sqrt{-2 \ln a_{t}} \times x_{i}
$$

Else

$$
\text { Get } y_{i}^{\prime}=E n+\sqrt{-2 \ln a_{t}} \times x_{i}
$$

\section{End If}

\section{End If}

$$
\text { If }\left(\mu_{i}^{\prime} \leqslant \mu_{\text {limit }}\right)
$$$$
\text { set length }=\text { length }-1
$$$$
\text { Set } \mu_{i}^{\prime}=0
$$

20) Get $y=y+\mu_{i}^{\prime} * y_{i}^{\prime}$

21) End While

22) Get $a_{t+1}=\frac{y}{\text { length }} \times\left(a_{\max }-a_{\min }\right)+a_{\min }$

\subsection{Multi-dimensional CM-MC}

Taking more than one factor into account can make load prediction for servers more accurate [24]. Therefore, we optimize the CM-MC based on multi-dimensional factors, including both CPU and RAM. Considering the connections between the multiple factors, the clouds in the two CMs $C_{a}\left\{c_{a_{1}}, c_{a_{2}}, \ldots, c_{a_{n}}\right\}$ and $C_{b}\left\{c_{b_{1}}, c_{b_{2}}, \ldots, c_{b_{n}}\right\}$ are numbered to get the state sets $\left\{J_{a}(1), J_{a}(2), \ldots, J_{a}(n)\right\}$ and $J_{b}\left\{J_{b}(1), J_{b}(2), \ldots, J_{b}(n)\right\}$. The cross-state set

$$
\left\{\begin{array}{cccc}
J_{a}(1) J_{b}(1) & J_{a}(1) J_{b}(2) & \cdots & J_{a}(1) J_{b}(m) \\
J_{a}(2) J_{b}(1) & J_{a}(2) J_{b}(2) & \cdots & J_{a}(2) J_{b}(m) \\
\vdots & \vdots & \ddots & \vdots \\
J_{a}(n) J_{b}(1) & J_{a}(n) J_{b}(2) & \cdots & J_{a}(n) J_{b}(m)
\end{array}\right\}
$$

is then obtained, which can also be expressed as vector $\left(J_{a}(1) J_{b}(1), J_{a}(1) J_{b}(2), \ldots, J_{a}(1) J_{b}(m), J_{a}(2) J_{b}(1)\right.$, $\left.\ldots, J_{a}(n) J_{b}(m)\right)$, to get the Markov state transition matrix $\boldsymbol{W}$. The membership degree vector is also upgraded. Taking $\mu_{a}=\left(\mu_{a_{1}}, \mu_{a_{2}}, \ldots, \mu_{a_{k}}\right)$ of $C_{a}\left\{c_{a_{1}}, c_{a_{2}}, \ldots\right.$, $\left.c_{a_{n}}\right\}$ as an example, the workflow is as follows.

Step 1 Set $i=0$.

Step 2 If $i<m \times n$, go to Step 3; otherwise, go to Step 5.

Step 3 Take the value of $r$ in $J_{a}(r)$ from $T$.

Step 4 Set $\mu_{a}^{\prime}[i]=\mu_{a_{r}}$, and go back to Step 2;

Step 5 The end.

In this way, the upgraded membership matrix of two CMs is obtained. The historical data at each point in time is recorded as $\left(a_{t}, b_{t}\right)$, where $a_{t}$ and $b_{t}$ represent the measured values at time $t$, and $a_{\max }$ and $a_{\min }$ represent the maximum and minimum values of the history training $a$ samples, while $b_{\max }$ and $b_{\min }$ represent the maximum and minimum values in the history training $b$ samples. $\left\{C_{a}\left(E x_{a_{i}}, E n_{a_{i}}, H e_{a_{i}}\right)(i=1,2, \ldots, n)\right\}$ and $\left\{C_{b}\left(E x_{b_{i}}, E n_{b_{i}}, H e_{b_{i}}\right)(i=1,2, \ldots, m)\right\}$ are obtained respectively according to their historical data. And then the prediction value $\left(a_{t+1}, b_{t+1}\right)$ is calculated synthetically based on the current time series $\left\{\left(a_{t-1}, b_{t-1}\right),\left(a_{t}, b_{t}\right)\right\}$ and the Markov transition matrix $\boldsymbol{T}$ is obtained. The pseudo-code is as follows.

Input: $\mathrm{CM}$ series $\left\{C_{a}\left(E x_{a_{i}}, E n_{a_{i}}, H e_{a_{i}}\right)(i=1\right.$, $2, \ldots, n)\} ; \mathrm{CM}\left\{C_{b}\left(E x_{b_{i}}, E n_{b_{i}}, H e_{b_{i}}\right)(i=1,2, \ldots\right.$, $m)\} ;\left\{\left(a_{t-1}, b_{t-1}\right),\left(a_{t}, b_{t}\right)\right\} ;$ Markov transfer matrix $\boldsymbol{W}$

Initialize: int $a_{t+1}=0$; int $b_{t+1}=0$; int $y_{1}=0$; int $y_{2}=0$

Output: Predictive value $\left(a_{t+1}, b_{t+1}\right)$

1) Set length $=m * n$

2) For $i$ from 0 to $n-1$

3) For $j$ from 1 to $m$

4) Get $\lambda_{i}=\mathrm{e}^{\left(-\left(\frac{a_{t}-E x_{-} C_{i}}{2 E n_{-} C_{i}}\right)^{2}\right)}$

5) $\quad \operatorname{Get} \beta_{j}=\mathrm{e}^{\left(-\left(\frac{b_{t}-E x_{-} R_{j}}{2 E n_{-} R_{j}}\right)^{2}\right)}$

6) End For

7) End For

8) Trans $(\lambda, \beta) \quad$ /* Handle the membership */

9) Get $\boldsymbol{P}(0)_{a}=\left(\mu_{a_{1}}, \mu_{a_{2}}, \ldots, \mu_{a_{\text {length }}}\right)$

10) Get $\boldsymbol{P}(0)_{b}=\left(\mu_{b_{1}}, \mu_{b_{2}}, \ldots, \mu_{b_{\text {length }}}\right)$

11) Get $\boldsymbol{P}(n)_{a}=\boldsymbol{P}(0)_{a} \boldsymbol{P}(n)=\left(\mu_{a_{1}}^{\prime}, \mu_{a_{2}}^{\prime}, \ldots, \mu_{a_{\text {length }}}^{\prime}\right)$

12) Get $\boldsymbol{P}(n)_{b}=\boldsymbol{P}(0)_{b} \boldsymbol{P}(n)=\left(\mu_{b_{1}}^{\prime}, \mu_{b_{2}}^{\prime}, \ldots, \mu_{b_{\text {length }}}^{\prime}\right)$

13) While (length)

14) If $\left(\mu_{a_{i}}^{\prime}>\mu_{\text {limit }}\right) \quad / * i=1,2,3,4, \ldots$, length $* /$

15) Get $z_{1}=\operatorname{normrnd}\left(E n_{a_{i}}, H e_{a_{i}}\right) \quad / *$ Generate a normal random number with expectation $E n_{a_{i}}$ of entropy $H e_{a_{i}} *$ /

16) If $\left(a_{t}>a_{t-1}\right)$

17) Get $y_{1}^{\prime}=E n_{a_{i}}+\sqrt{-2 \ln \left(a_{t}\right)} \times z_{1}$

18) Else

19) Get $y_{1}^{\prime}=E n_{a_{i}}-\sqrt{-2 \ln \left(a_{t}\right)} \times z_{1}$

20) End If

21) End If

22) If $\left(\mu_{a_{i}}^{\prime} \leqslant \mu_{\text {limit }}\right)$

23) Set $n=n-1$

24) $\quad$ Set $\lambda_{i}=0$

25) End If

26) If $\left(\mu_{b_{i}}^{\prime}>\mu_{\text {limit }}\right) \quad / * i=1,2,3,4, \ldots$, length $* /$

27) Get $z_{2}=\operatorname{normrnd}\left(E n_{b_{i}}, H e_{b_{i}}\right) \quad / *$ Generate a normal random number with expectation $E n_{b_{i}}$ of entropy $\mathrm{He}_{b_{i}} * 1$

28) If $\left(b_{t}>b_{t-1}\right)$

29) $\quad$ Get $y_{2}^{\prime}=E n_{b_{i}}+\sqrt{-2 \ln \left(b_{t}\right)} \times z_{2}$

30) Else

31) $\quad$ Get $y_{2}^{\prime}=E n_{b_{i}}-\sqrt{-2 \ln \left(b_{t}\right)} \times z_{2}$

32) End If 

33) End If
34) If $\left(\mu_{a_{i}}^{\prime} \leqslant \mu_{\text {limit }}\right)$
35) Set $m=m-1$
36) $\quad$ Set $\beta_{i}=0$
37) End If
38) Get $y_{1}=y_{1}+\lambda_{i} * y_{1}^{\prime}$
39) Get $y_{2}=y_{2}+\beta_{i} * y_{2}^{\prime}$
40) End While
41) Get $a_{t+1}=\frac{y_{1}}{n} \times\left(a_{\max }-a_{\min }\right)+a_{\min }$
42) Get $b_{t+1}=\frac{y_{2}}{m} \times\left(b_{\max }-b_{\min }\right)+b_{\min }$

\section{Experiments and analysis}

\subsection{Experimental platform}

We build the mixed experimental platform based on the real distributed computing cluster and the simulation tool, and implemented a series of experiments to test and compare the performances of the prediction algorithms based on CM-MC with the BP neural network, the Markov process and the CM. The historical data for model training and the data for comparison experiments are the true measured values of CPU and RAM utilization under the pressure test.

We use the OpenStack, CentOS and the PHPC100 highperformance server cluster to build the basic cloud computing system.

The experiment includes four steps.

(i) The above algorithms are under stress tests to compare with each other for the CPU utilization prediction.

(ii) These algorithms are under stress tests to compare with each other for the memory utilization prediction.

(iii) The average absolute value of relative error between the predicted and actual results are compared to verify the prediction accuracy of these algorithms.

Set the predicted value as $w_{t}$ and the real data as $c_{t}$ at time $t$. The prediction results of $n$ rounds are calculated, and then the average absolute value of relative error $\delta$ between prediction data and real data is obtained, with

$$
\delta=\frac{\sum_{t=1}^{n}\left|\frac{c_{t}-w_{t}}{c_{t}}\right|}{n} .
$$

\subsection{Experiments}

Fig. 4 shows the prediction results of CPU utilization. As Fig. 4 shows, compared with the prediction algorithms based on the $\mathrm{CM}$, the $\mathrm{MC}$, or the BP neural network, our prediction algorithm based on CM-MC is more suitable for situations with high load volatility. Both the prediction algorithms based on the $\mathrm{CM}-\mathrm{MC}$ and the $\mathrm{CM}$ can forecast the fluctuation of load in time, while the prediction algorithm based on CM-MC can achieve the better prediction accuracy. Compared with the prediction algorithm based on the MC, the prediction algorithm based on CM-MC is more suitable for situations with high load volatility and its prediction is more accurate. The problem of the prediction algorithm based on the BP neural network is that its prediction needs many iterations and its prediction accuracy is lower than our prediction algorithm based on CM-MC.

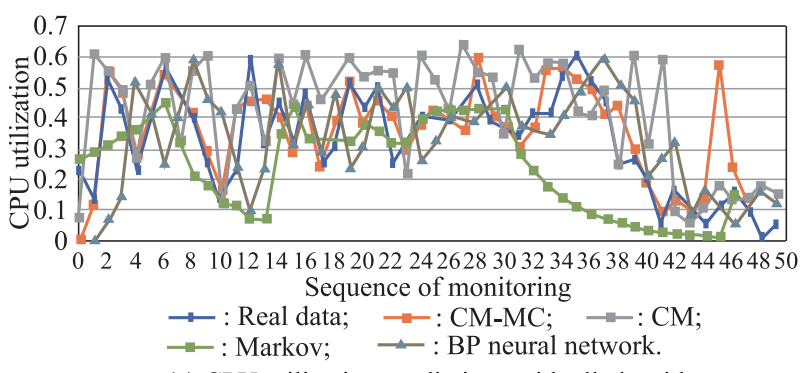

(a) CPU utilization predictions with all algorithms

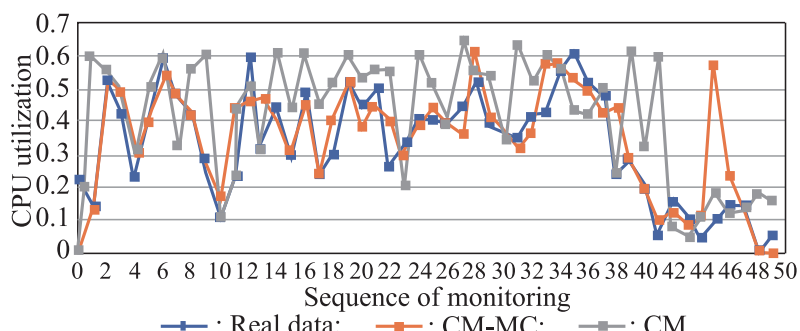

(b) $\mathrm{CPU}$ utilization predictions with $\mathrm{CM}-\mathrm{MC}$ and the $\mathrm{CM}$

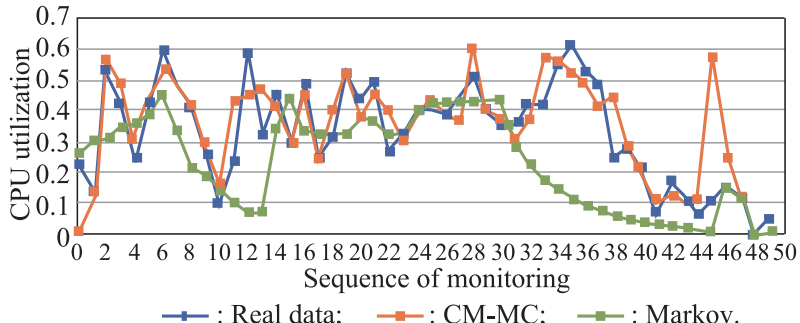

(c) CPU utilization predictions with CM-MC and Markov

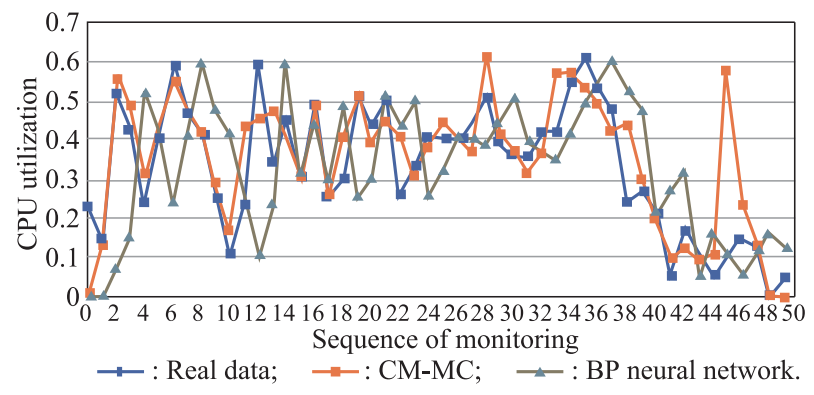

(d) CPU utilization predictions with CM-MC and the BP neural network

Fig. 4 Predictions of CPU utilization with different algorithms

Fig. 5 shows the absolute value of average relative error of CPU utilization between the forecast data and real loads. 


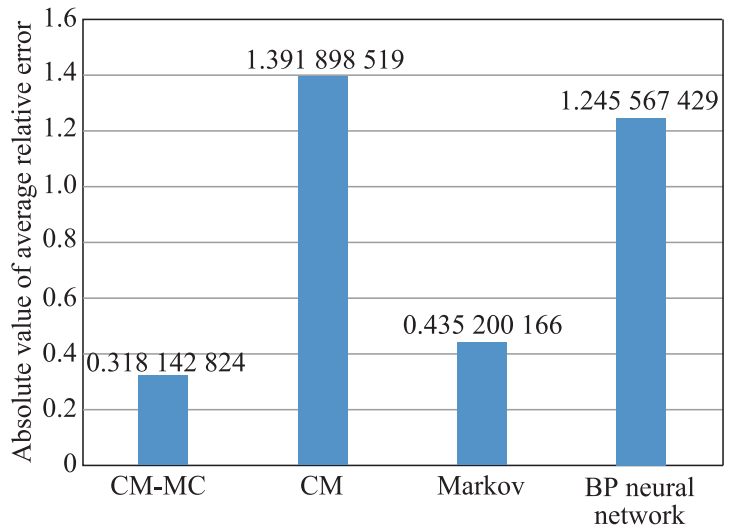

Fig. 5 Absolute values of average relative error of CPU utilization

It can be seen that the absolute values of average relative error of both the Markov-based prediction algorithm and the CM-based prediction algorithm are much higher than those of our algorithm. The absolute value of average relative error of the Markov-based prediction algorithm is closest to our algorithm, while it is not sensitive to the fluctuation of load.

Fig. 6 shows the prediction results of RAM utilization. It can be seen that for the volatility of load is not very strong, the performance of our algorithm CM-MC has the weak advantage in the prediction accuracy.

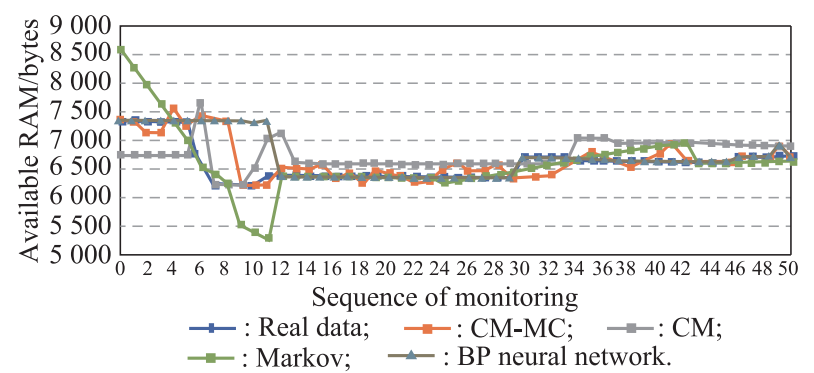

(a) RAM utilization predictions with all algorithms

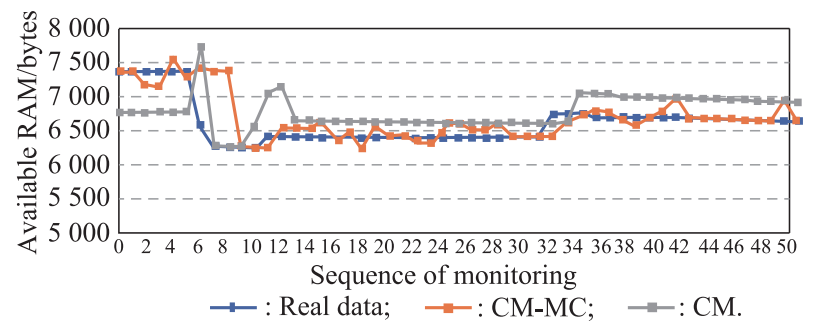

(b) RAM utilization predictions with $\mathrm{CM}-\mathrm{MC}$ and $\mathrm{CM}$

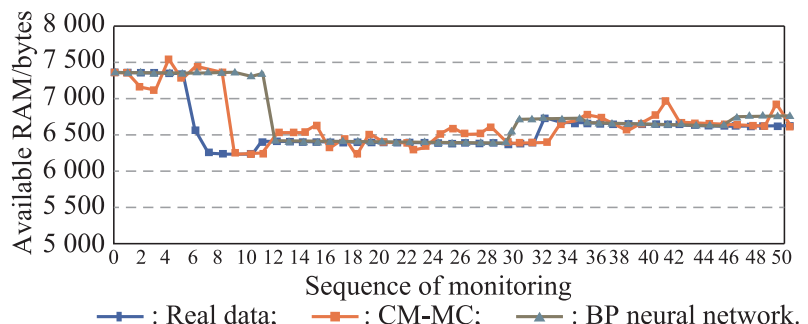

(c) RAM utilization predictions with CM-MC and BP neural network

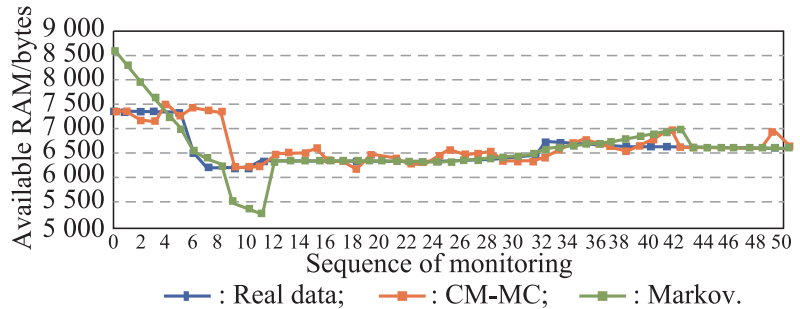

(d) RAM utilization predictions with CM-MC and BP Markov

Fig. 6 Predictions of RAM utilization with different algorithms

Fig. 7 shows the absolute value of average relative error of forecast results and real data of available RAM.

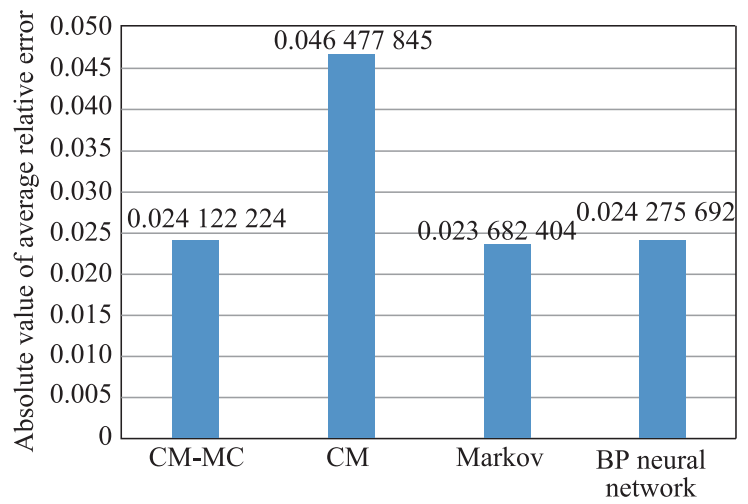

Fig. 7 Average absolute value of relative error of available RAM

It can be seen that the absolute value of average relative error of the prediction algorithm based on the CM is higher than other algorithms, and the prediction accuracy of other algorithms, including our algorithm, are quite close to each other. It can be seen that the prediction algorithm based on CM-MC shows very good performance for non-linear and highly volatile loads from the experimental results.

\subsection{Computational complexity}

CM-MC utilizes the historical data to establish the CM, and then predict the load according to the current state. Because of the timeliness problem, the CM does not need to be modified for a period of time. The time complexity of the model training is $O\left(n^{2}\right)$, and the time complexity of the prediction is $O(n)$. Compared with the non-heuristic algorithms, such as the Markov-based prediction algorithm, the prediction accuracy of the CM-MC algorithm has obvious advantage. Compared with the heuristic algorithms, such as the prediction algorithm based on BP neural network, whose time complexity of training a BP neural network or prediction is $O\left(n^{2}\right)$, the time complexity of our algorithm is lower, and the forecast time is reduced.

\section{Conclusions}

The load prediction is very important and means a lot to the green cloud computing. In this paper, we combine the 
$\mathrm{CM}$ and Markov process to propose a new server load prediction algorithm. The algorithm is applied to cloud systems and suitable for predicting load with high volatility. Compared with other typical algorithms, our algorithm not only can achieve better prediction accuracy, but also has lower calculation cost. The prediction granularity of the algorithm is suitable for the real-time server load prediction for cloud systems, which is important to reduce the energy consumption caused by the virtual machine migration and improve the performance of virtual machine migration. In the future, we plan to put more factors into consideration besides CPU and RAM. The main problem is that with more factors, the storage of the MC needs to be improved with introducing the sparse matrix, etc.

\section{References}

[1] CALHEIROS R, MASOUMI E, RANJAN R, et al. Workload prediction using ARIMA model and its impact on cloud applications' QoS. IEEE Trans. on Cloud Computing, 2015, 3(4): $449-458$.

[2] BARATI M, SHARIFIAN S. A hybrid heuristic-based tuned support vector regression model for cloud load prediction. The Journal of Supercomputing, 2015, 71(11): 4235-4259.

[3] JIANG Y X, PENG C, LI T, et al. Asap: A self-adaptive prediction system for instant cloud resource demand provisioning. Proc. of the 11th IEEE International Conference on Data Mining, 2011: $1104-1109$.

[4] KHAN A, YAN X, TAO S, et al. Workload characterization and prediction in the cloud: a multiple time series approach. Proc. of the IEEE International Conference on Network Operations and Management Symposium, 2012: 1287-1294.

[5] LIU X Y. Research and implementation of virtual server consolidation based on forecasting in cloud environment. Nanjing, China: Nanjing University of Posts and Telecommunications, 2015.

[6] POHLKER M L, POHLKER C, DITAS F, et al. Long-term observations of cloud condensation nuclei in the Amazon rain forest-Part 1: Aerosol size distribution, hygroscopicity, and new model parametrizations for $\mathrm{CCN}$ prediction. Atmospheric Chemistry and Physics, 2016, 16(24): $15709-15740$.

[7] ZHANG B B, CHEN N J, HU D D. Virtual machine deployment strategy by load prediction based on BP neural network. Journal of Huazhong University of Science and Technology, 2012, 40: $120-123$.

[8] LIU L X, MEI H, XIE B. Towards a multi-QoS human-centric cloud computing load balance resource allocation method. The Journal of Supercomputing, 2016, 72(7): 2488-2501.

[9] KALYVIANAKI E, HAND S. Applying Kalman filters to dynamic resource provisioning of virtualized server applications. Proc. of the 3rd International Workshop on Feedback Control Implementation and Design in Computing System and Networks, 2008: 1-6.

[10] MUNOZ-ESCOI F D, BERNABEU-AUBAN J M. A survey on elasticity management in PaaS systems. New York: Springer-Verlag, 2017.

[11] LI P H, LI Y G, XIONG Q Y, et al. Application of a hybrid quantized Elman neural network in short-term load forecasting. International Journal of Electrical Power \& Energy Systems, 2014, 55(2): 749-759.

[12] HAO Y S, WANG L N, ZHENG M. An adaptive algorithm for scheduling parallel jobs in meteorological cloud. KnowledgeBased Systems, 2016, 98(C): 226-240.
[13] YANG C H, LI Y D. Planar model and its application in prediction. Chinese Journal of Comupters, 1998, 21(11): 961 - 969.

[14] CAO J, FU J W, LI M L, et al. CPU load prediction for cloud environment based on a dynamic ensemble model. SoftwarePractice and Experience, 2014, 44(7): 793 - 804.

[15] HAMEED A, KHOSHKBARFOROUSHHA A, RANJAN R, et al. A survey and taxonomy on energy efficient resource allocation techniques for cloud computing systems. Computing, 2016, 98(7): 1-24.

[16] SHENG Z, XIE S Q, PAN C Y. Probability theory and mathematical statistics. Beijing, China: Higher Education Press, 2009.

[17] CHEN J B, PENG Y J, ZHI X F, et al. Research on application classification method in cloud computing environment. Proc. of the 2nd IEEE International Conference on Cyber Security and Cloud Computing, 2015: 524-529.

[18] LI D Y, LIU C, GAN W. A new cognitive model: cloud model. International Journal of Intelligent Systems, 2010, 24(3): $357-375$.

[19] LIU C Y, LI Y D, DU Y, et al. Some statistical analysis of the normal cloud model. Information and Control, 2005, 34(4): $236-239$.

[20] JIN L. Application research of the cloud model in time series prediction. Chengdu, China: University of Electronic Science and Technology of China, 2014

[21] LI D Y. Uncertainty in knowledge representation. Engineering Science, 2000, 2(10): $73-79$.

[22] MENG H, WANG S L, LI D Y. Concept extraction and concept hierarchy construction based on cloud transformation. Journal of Jilin University, 2010, 40(3): $782-787$.

[23] ZHA X, NI S H, XIE C, et al. Indirect computation approach of cloud model similarity based on conception skipping. Systems Engineering and Electronics, 2015, 37(7): 1676-1682. (in Chinese)

[24] LI W B, LI Z H, CHEN Q, et al. Inconsistencies detection in distributed big data. Journal of Software, 2016, 27(8): 2068 2085 .

\section{Biographies}

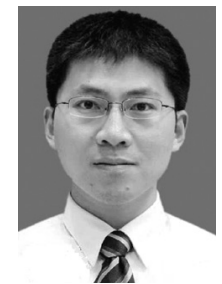

XU Xiaolong was born in 1977. He received his B.S. degree in computer and its applications, M.S. degree in computer software and theories and Ph.D. degree in communications and information systems from Nanjing University of Posts \& Telecommunications, Nanjing, China, in 1999, 2002 and 2008, respectively. He worked as a postdoctoral researcher at Station of Electronic Science and Technology, Nanjing University of Posts \& Telecommunications from 2011 to 2013. $\mathrm{He}$ is currently a professor in College of Computer, Nanjing University of Posts \& Telecommunications. He is a senior member of China Computer Federation. His current research interests include cloud computing, mobile computing, intelligent agent and information security.

E-mail: xuxl@njupt.edu.cn

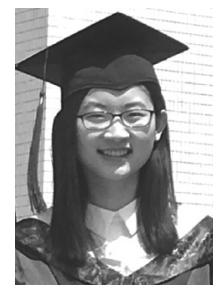

ZHANG Qitong was born in 1992. She received her B.E. degree in software engineering from Nanjing University of Posts \& Telecommunications, Nanjing, China, in 2014. She is now a postgraduate student majoring in software engineering, and working in a project team supported by the State Key Laboratory of Information Security, Chinese Academy of Sciences, Beijing, China. Her current research interests include cloud computing and virtualization.

E-mail: 1014041119@njupt.edu.cn 


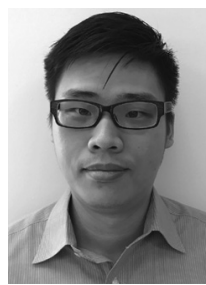

MOU Yiqi was born in 1989. He received his B.E. degree in network engineering from Nanjing University of Posts \& Telecommunications, Nanjing, China, in 2011, and M.E. degree in computer technology from Nanjing University of Posts \& Telecommunications, Nanjing, China, in 2016, respectively. He works as an engineer in Data Center Business Department at Shanghai Stock Exchange Technology Co., Ltd., Shanghai, China, carrying out research in cloud computing and data center network technology.

E-mail:yqmou@sse.com.cn

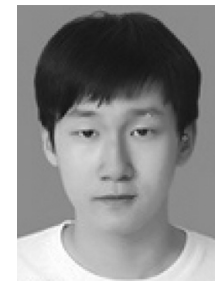

LU Xinyuan was born in 1995. He received his B.E. degree in computer science and technology from Nanjing University of Posts \& Telecommunications, Nanjing, Nanjing, China, in 2017. He works as an engineer in Institute of Big Data Research at Yancheng, Nanjing University of Posts and Telecommunications, Yancheng, China, carrying out research in cloud computing and data analysis.

E-mail: 1016041226@njupt.edu.cn 